\title{
New findings on the dynamics of HIV and TB coinfection models
}

\author{
Carla M.A. Pinto, Ana R.M. Carvalho
}

\begin{abstract}
In this paper we study a model for HIV and TB coinfection. We consider the integer order and the fractional order versions of the model. Let $a 2 \%: 78 ; 1: 0$ ] be the order of the fractional derivative, then the integer order model is obtained for $a 1 / 41: 0$. The model includes vertical transmission for HIV and treatment for both diseases. We compute the

reproduction number of the integer order model and HIV and TB submodels, and the sta- bility of the disease free equilibrium. We sketch the bifurcation diagrams of the integer order model, for variation of the average number of sexual partners per person and per unit time, and the tuberculosis transmission rate. We analyze numerical results of the fractional order model for different values of $a$, including $a 1 / 41$. The results show distinct types of

transients, for variation of $a$. Moreover, we speculate, from observation of the numerical

results, that the order of the fractional derivative may behave as a bifurcation parameter for the model. We conclude that the dynamics of the integer and the fractional order versions of the model are very rich and that together these versions may provide a better understanding of the dynamics of HIV and TB coinfection.
\end{abstract}

Keywords:

HIV, Tuberculosis (TB), Coinfection, Integer model, Fractional model

\section{Introduction}

Tuberculosis (TB) is an infectious disease caused by the bacteria Mycobacterium tuberculosis (MTB). It is transmitted through droplet transmission, when people suffering from pulmonary TB expel bacteria, for example, by coughing. In general, a relatively small proportion of people infected with MTB develops active TB, but this proportion is much higher in people with impaired immunity.

TB is a major health problem worldwide. Annually, 9 million of new TB cases are estimated throughout the globe. TB is particularly severe in certain populations, since it appears associated with poverty and the syndrome of immunodeficiency virus (HIV)/AIDS coinfection. In 1993, the World Health Organization (WHO) declared TB a global emergency by its overall size and consequences, namelly its association with HIV/AIDS, and the emergence of multidrug-resistance. The Global Plan to Stop TB [28] indicates the goals to be achieved in the 'war' against TB. Some of these goals are to determine strategies and costs associated with multidrug-resistant TB, reduce consequences of coinfection with HIV, to aid laboratories to develop research on TB in a consistent and coordinated way, amongst others.

Treatment of TB is achieved through antibiotics. Treatment takes at least six months and is increasingly hampered by multidrug-resistance. There are 6 types of multidrug-resistant TB [28]. The treatment for TB has had a success rate of $87 \%$ worldwide. In EU/EEA this success rate drops to 74.3\% [7]. Treatment success had been achieved in $76.8 \%$ of new pulmonary 

TB cases, $53.7 \%$ of previously treated cases, and 31.6\% of multidrug-resistant TB cases. Some first-line drugs used to treat TB are rifampicin, isoniazid,pyrazinamide, ethambutol. The second line drugs includes amikacin, capreomycin, cycloserine, azithromycin, clarithromycin, moxifloxacin, levofloxacin. The exposed individuals are treated with isoniazid.

The human immunodeficiency virus (HIV) is a retrovirus that causes acquired immunodeficiency syndrome (AIDS), characterized as progressive failure of the immune system. AIDS leaves the human body vulnerable to life-threatening opportunistic infections and cancers. The modes of transmission include unprotected sexual intercourse, sharing of needles and syringes for injecting drugs, mother-to-child transmission, and the transfusion of contaminated blood or its products.

HIV treatment is based on the use of antiretroviral drugs (ART), to reduce its spread and AIDS-related mortality. These drugs act on the body slowing down HIV replication. It is recommended, usually three or more drugs to reduce viral load [27]. In what concerns vertical transmission (from mother to child) it is known that an HIV-positive woman has a 50\% chance of infecting her child [3].

HIV and MTB have a somewhat synergistic relationship. The HIV/AIDS pandemic plays an important role in the resurgence of TB, increasing morbidity and mortality worldwide. It decreases the TB latency period, accelerating active disease in people infected with MTB. Namely, an HIV-positive person infected with MTB has a $50 \%$ chance of developing active TB, whereas an HIV-negative person has only a $10 \%$ chance of developing it [12]. The statistics of the two diseases show that, in 2010, 2.7 million people were infected with HIV and about 8.8 million were infected with TB. Of these, $13 \%$ were infected with HIV. The number of TB deaths associated with HIV was estimated on 1.1 million and 0.35 million HIV-negative people died from TB in 2010.

In this paper, we propose a model for HIV and TB coinfection. We consider two versions of the model, the integer order and the fractional order versions. The order of the fractional derivative is $a$, and for $a 1 / 41$, the fractional order version reduces to the integer order one. We consider vertical transmission from mother to child and treatment for both diseases. In Section 2 , we describe the two versions of the model, compute the reproduction number and the local stability of the disease free equilibrium. We also sketch some bifurcation diagrams for the integer order version of the model. In Section 3 , we analyze numerical simulations of the fractional order model, for different values of the order of the fractional derivative, $a$. Finally, in Section 4 , we state the conclusions of our work.

\subsection{Fractional calculus - brief summary}

Fractional calculus (FC) $[15,23,10,24]$ has received increased attention by mathematicians and engineers, in the last few decades. Important applications of FC are, for example, in fluid mechanics [11], electrochemistry [14], engineering [9,25,22,26,1,21,20], physics [13,2], and biology [16-19].

The definition of fractional order or non-integer order derivative is not unique. There are three important and well studied definitions, namely, the Riemann-Liouville, the Grünwald-Letnikov, and the Caputo formula [10,15]. We will focus on the Grünwald-Letnikov (GL) derivative here, given by the following equation:

$a$

$$
{ }_{a}^{C .} D_{t}^{\alpha} f(t)=\lim _{h \rightarrow 0} \frac{1}{h^{\alpha}} \sum_{k=0}^{[-a]}(-1)^{k}\left(\begin{array}{l}
\alpha \\
k
\end{array}\right) f(t-k h), \quad t>a, \quad \alpha>0,
$$

where $1 / 2 x]$ means the integer part of $x$, and $h$ represents the time step increment.

These definitions outline an important characteristic of fractional derivatives: they capture the history of the variable, or, by other words, that have memory, contrary to integer order derivatives, that are local operators. This characteristic makes them attractive in the modeling of memory-intense and delay systems.

The Grünwald-Letnikov formulation inspires a discrete-time algorithm, based on the approximation of the time increment $h$, through the sampling period $T$, and the series truncation at the $r$ th order. This method is often denoted as Power Series Expansion (PSE) yielding the following equation in the $z$ - domain:

$$
\frac{Z\left\{D^{\alpha} f(t)\right\}}{\mathcal{Z}\{f(t)\}}=\frac{1}{T^{2}} \sum_{k=0}^{\infty} \frac{(-1)^{k} \Gamma(\alpha+1)}{k ! \Gamma(\alpha-k+1)} z^{k}=\left(\frac{1-z^{-1}}{T}\right)^{\alpha},
$$

where $X \succsim z \mathrm{P} 1 / 4 \mathrm{Z} \mathbf{f} x \partial \mathrm{t} \mathrm{Pg}$ and $z$ and $Z$ represent the $z$-transform variable and operator, respectively. The corresponding truncated series of $r$ terms is:

$$
\frac{\left.Z D^{\alpha} f(t)\right\}}{\mathcal{Z}\{f(t)\}}=\frac{1}{T^{2}} \sum_{k=0}^{r} \frac{(-1)^{k} \Gamma(\alpha+1)}{k ! \Gamma(\alpha-k+1)} z^{-k}
$$

Infact, expression (3) represents the Euler (or first backward difference) approximationin thes ! $z$ discretisation scheme, being the Tustin approximation another possibility.

\section{Description of model}

The model for HIV and TB coinfection presented here, includes vertical transmission for HIV/AIDS and treatment for both diseases $[27,4,8]$. 
The entire population is divided in five classes: the susceptible individuals, SðtP, the HIV infected individuals, I $t \mathrm{P}$, the HIV infected, showing symptoms of AIDS, individuals, AðtP, the TB infected individuals, $I_{T} \circlearrowright t \mathrm{P}$, and the dually HIV and TB infected individuals, showing or not symptoms of AIDS, $I_{H T}$ ठtP. We assume that individuals with AIDS, A, and dually infected individuals, $I_{H T}$, are aware of their condition and develop suitable precautionary measures, so that they cannot infect anyone else.

The population of size $N \delta t \mathrm{P}$, at time $t$, has inflow, $p N$, of susceptibles, SðtP, where $p$ is the recruitment rate. The natural mortality rate is $\boldsymbol{l}$ in all classes. Susceptible individuals, $S$, are infected with HIV, at a rate $\frac{c_{1} b_{1} I S}{N}$. They can acquire TB at a rate $\frac{b I_{T} \underline{S}}{N}$, and move to class $I_{T}$ ठtP. Parameter $b_{1}$ is the sexual contact rate, $c_{1}$ is the average number of sexual partners per person and per unit time, and $b$ is the tuberculosis transmission rate.

A fraction of new born children are infected during birth and hence directly recruited into the infectious classes, Ið $\mathrm{t} \mathrm{P}$, at a rate 01 - EPh. Other children die at birth $006 \mathrm{E} 6$ 1P, where $\mathrm{E}$ is the fraction of newborns infected with HIV who dies immediately after birth, and $h$ is the rate of newborns infected with HIV. Infected HIV individuals, IठtP, move, at a rate $d$, to the AIDS class, $A$. They may also progress to class $I_{H T} \circlearrowright t \mathrm{P}$, by acquiring TB, at a rate $\frac{b_{3} I_{T}}{N}$, where $b_{3} \mathbf{P} b$. Note that TB is an airborne trans ${ }^{-}$ mitted disease, so HIV infected individuals with lowered immunity system are more susceptible to acquire and develop TB. Individuals with AIDS, $A$, are treated at a rate $m$, and die because of AIDS at a rate $d_{1}$.

Individuals infected with TB, $I_{T}$ ठtP, are assumed to take enough care so that they are not infected with other diseases, so there is no HIV coinfection. They can be treated for TB and move to the susceptible class at a rate $t_{2}$, or die at a rate $d_{2}$.

Dually infected with HIV/AIDS and TB individuals, $I_{T H} \circlearrowright t \mathrm{P}$, are treated for TB and move to the IठtP class, at a rate $t_{1}$. They die from the two diseases at a rate $d_{3}$. Note that $d_{3}>d_{2} \mathrm{p} d_{1}$, due to more severity of the diseases in the case of coinfection.

The schematic diagram of the proposed model can be found in Fig. 1.

The integer order system of ordinary differential equations for the proposed model is:

$$
\begin{aligned}
& \frac{d S}{d t}=\pi N+t_{2} I_{T}-\frac{c_{1} \beta_{1} I S}{N}-\frac{\beta I_{T} S}{N}-\mu S, \\
& \frac{d l}{d t}=\frac{c_{1} \beta_{1} I S}{N}+v A+t_{1} I_{H T}+(1-\epsilon) \theta I-\frac{\beta_{3} I_{T} I}{N}-(\delta+\mu) I, \\
& \frac{d A}{d t}=\delta I-\left(v+d_{1}+\mu\right) A, \\
& \frac{d l_{T}}{d t}=\frac{\beta S I_{T}}{N}-\left(\mu+d_{2}+t_{2}\right) I_{T}, \\
& \frac{d l_{T H}}{d t}=\frac{\beta_{3} I_{T} I}{N}-t_{1} I_{H T}-\left(d_{3}+\mu\right) I_{T H} .
\end{aligned}
$$

The dynamics of the total population $N(t)=S(t)+I(t)+A(t)+I_{T}(t)+I_{\text {TH }}(t)$, is given by:

$$
\frac{d N}{d t}=(\pi-\mu) N+(1-\epsilon) \theta I-d_{1} A-d_{2} l_{T}-d_{3} I_{T H} .
$$

The variables can be normalized as follows: $s=\frac{S}{N}, i=\frac{1}{N}, a=\frac{A}{N}, i_{T}=\frac{L_{N}}{N}$ and $i_{T H}=\frac{L^{N}}{N}$. The normalized system is given by:

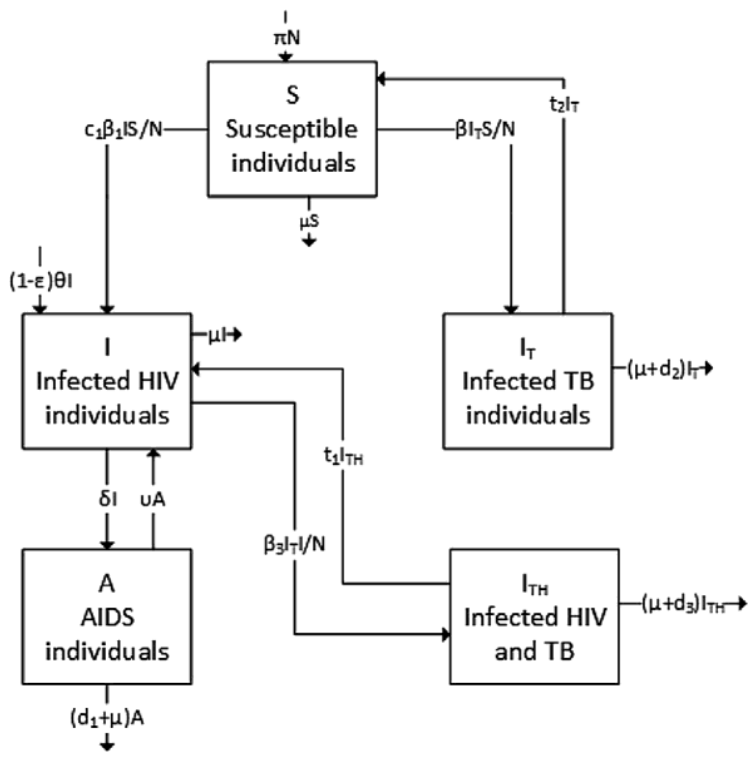

Fig. 1. Schematic diagram of the fractional order model (4). 


$$
\begin{aligned}
& \frac{d s}{d t}=\pi+t_{2} i_{T}-c_{1} \beta_{1} i s-\beta i_{T} s-s\left(\pi+(1-\epsilon) \theta i-d_{1} a-d_{2} i_{T}-d_{3} i_{T H}\right), \\
& \frac{d i}{d t}=c_{1} \beta_{1} i s+v a+t_{1} i_{H T}+(1-\epsilon) \theta i-\beta_{3} i_{T} i-\delta i-i\left(\pi+(1-\epsilon) \theta i-d_{1} a-d_{2} i_{T}-d_{3} i_{T H}\right), \\
& \frac{d a}{d t}=\delta i-\left(v+d_{1}\right) a-a\left(\pi+(1-\epsilon) \theta i-d_{1} a-d_{2} i_{T}-d_{3} i_{T H}\right), \\
& \frac{d i_{T}}{d t}=\beta s i_{T}-\left(d_{2}+t_{2}\right) i_{T}-i_{T}\left(\pi+(1-\epsilon) \theta i-d_{1} a-d_{2} i_{T}-d_{3} i_{T H}\right), \\
& \frac{d i_{T H}}{d t}=\beta_{3} i_{T} i-t_{1} i_{H T}-d_{3} i_{T H}-i_{T H}\left(\pi+(1-\epsilon) \theta i-d_{1} a-d_{2} i_{T}-d_{3} i_{T H}\right) .
\end{aligned}
$$

The corresponding fractional (non-integer) order system is given by:

$$
\begin{aligned}
& \frac{d s^{\alpha_{1}}}{d t}=\pi+t_{2} i_{T}-c_{1} \beta_{1} i s-\beta i_{T} s-s\left(\pi+(1-\epsilon) \theta i-d_{1} a-d_{2} i_{T}-d_{3} i_{T H}\right), \\
& \frac{d t^{\alpha_{2}}}{d t}=c_{1} \beta_{1} i s+v a+t_{1} i_{H T}+(1-\epsilon) \theta i-\beta_{3} i_{T} i-\delta i-i\left(\pi+(1-\epsilon) \theta i-d_{1} a-d_{2} i_{T}-d_{3} i_{T H}\right), \\
& \frac{d a^{\alpha_{3}}}{d t}=\delta i-\left(v+d_{1}\right) a-a\left(\pi+(1-\epsilon) \theta i-d_{1} a-d_{2} i_{T}-d_{3} i_{T H}\right), \\
& \frac{d i_{T}^{\alpha_{4}}}{d t}=\beta s i_{T}-\left(d_{2}+t_{2}\right) i_{T}-i_{T}\left(\pi+(1-\epsilon) \theta i-d_{1} a-d_{2} i_{T}-d_{3} i_{T H}\right), \\
& \frac{d i_{T H}^{z_{S}}}{d t}=\beta_{3} i_{T} i-t_{1} i_{H T}-d_{3} i_{T H}-i_{T H}\left(\pi+(1-\epsilon) \theta i-d_{1} a-d_{2} i_{T}-d_{3} i_{T H}\right) .
\end{aligned}
$$

where $\left.a_{1} ; \ldots ; a_{5} 20: 78 ; 1: 0\right]$, is the order of the fractional derivative. For $a 1 / 41$, we obtain model (6).

\subsection{Reproduction numbers and stability of disease-free equilibria}

In this subsection, we compute the reproduction number, $R_{0}$, of model (6). The basic reproduction number is the number of secondary infections due to a single infection, in a completely susceptible population.

We begin by considering the following two sub-models of model (6). Model (6) is derived from model (6) by setting the variables concerning HIV dynamics $\left(i ; a\right.$ and $i_{T H}$ ) to zero. Model (10) follows from model (6) by setting the variables concerning TB dynamics $\left(i_{T}\right.$ and $\left.i_{T H}\right)$ to zero.

We begin by computing the reproduction number, $R_{T B}$, of system (6). We use the next generation method [5].

$$
\begin{aligned}
& \frac{d s}{d t}=\pi-\beta i_{T} s+t_{2} i_{T}-s\left(\pi-d_{2} i_{T}\right), \\
& \frac{d i}{d t}=\beta s i_{T}-\left(d_{2}+t_{2}\right) i_{T}-i_{T}\left(\pi-d_{2} i_{T}\right) .
\end{aligned}
$$

The disease-free equilibrium of model (6) is given by:

$$
P_{0}^{1}=\left(s_{0}^{1}, i_{T_{0}}^{1}\right)=(1,0)
$$

We now compute the stability of the disease-free equilibrium $P_{0}^{1}$ of the TB submodel (6). The jacobian matrix corresponding to system (6), around the disease free-equilibrium $P_{0}^{1}$, is given by:

$$
\text { I }
$$

$$
M=\left[\begin{array}{cc}
-\pi & -\beta+t_{2} \\
0 & \beta-\left(\pi+d_{2}+t_{2}\right)
\end{array}\right] .
$$

The two eigenvalues of matrix $M$ are easily obtained and are given by:

$$
-\pi, \quad \beta-\left(\pi+d_{2}+t_{2}\right) .
$$

For the disease-free equilibrium $P_{0}^{1}$ to be asymptotically stable, these eigenvalues must be negative. As such:

$$
\beta-\left(\pi+d_{2}+t_{2}\right)<0 \Longleftrightarrow \beta<\pi+d_{2}+t_{2} \Longleftrightarrow \frac{\beta}{\pi+d_{2}+t_{2}}<1 .
$$

The associative basic reproduction number is, thus, given by:

$$
R_{7 B}=\frac{\beta}{\pi+d_{2}+t_{2}}
$$

Lemma 1 [5]. The disease-free equilibrium $P_{0}^{1}$ is locally asymptotically stable if $R_{T B}<1$ and unstable if $R_{T B}>1$.

We proceed with the computation of the reproduction number, $R_{H I V}$, of model (10) given by: 


$$
\begin{aligned}
& \frac{d s}{d t}=\pi-c_{1} \beta_{1} i s-s\left(\pi+(1-\epsilon) \theta i-d_{1} a\right), \\
& \frac{d i}{d t}=c_{1} \beta_{1} i s+v a+(1-\epsilon) \theta i-\delta i-i\left(\pi+(1-\epsilon) \theta i-d_{1} a\right), \\
& \frac{d a}{d t}=\delta i-\left(v+d_{1}\right) a-a\left(\pi+(1-\epsilon) \theta i-d_{1} a\right) .
\end{aligned}
$$

The disease-free equilibrium state $P_{0}^{2}$ of model (10) is computed to be:

$$
P_{0}^{2}=\left(s_{0}^{2}, i_{0}^{2}, a_{0}^{2}\right)=(1,0,0) .
$$

Using the notation of [5], matrices for the new infection terms, $F$, and the other terms, $V$, are the following:

$$
F=\left[\begin{array}{cc}
c_{1} \beta_{1} & 0 \\
0 & 0
\end{array}\right], \quad V=\left[\begin{array}{cc}
\delta+\pi-(1-\epsilon) \theta & -v \\
-\delta & v+d_{1}+\pi
\end{array}\right] .
$$

The associative basic reproduction number is given by:

$$
R_{H V}=\rho\left(F V^{-1}\right)=\frac{\beta_{1} c_{1}\left(v+d_{1}+\pi\right)}{\pi^{2}+\pi\left(d_{1}+v+\delta-(1-\epsilon) \theta\right)+\left(d_{1} \delta-(1-\epsilon) \theta\left(v+d_{1}\right)\right)},
$$

where $q$ indicates the spectral radius of $F V^{-1}$. By Theorem 2 [5], we obtain the following lemma.

Lemma 2. The disease-free equilibrium $P_{0}^{2}$ is locally asymptotically stable if $R_{H I V}<1$ and unstable if $R_{H I V}>1$.

We now proceed with the calculation of the reproduction number, $R_{0}$, of the full model (4). The disease-free equilibrium state $P_{0}$ of model (4) is given by:

$$
P_{0}=\left(s_{0}, i_{0}, a_{0}, i_{T_{0}}, i_{T H_{0}}\right)=(1,0,0,0,0) \text {. }
$$

Using the notation in [5] on system (4), matrices for the new infection terms, $F$, and the other terms, $V$, are given by:

$$
\begin{aligned}
& F=\left(\begin{array}{cccc}
c_{1} \beta_{1} & 0 & 0 & 0 \\
0 & 0 & 0 & 0 \\
0 & 0 & \beta & 0 \\
0 & 0 & 0 & 0
\end{array}\right), \\
& V=\left(\begin{array}{cccc}
\delta+\pi-(1-\epsilon) \theta & -v & 0 & -t_{1} \\
-\delta & v+d_{1}+\pi & 0 & 0 \\
0 & 0 & \pi+d_{2}+t_{2} & 0 \\
0 & 0 & 0 & t_{1}+d_{3}+\pi
\end{array}\right) .
\end{aligned}
$$

The associative basic reproduction number is thus:

$$
R_{0}=\rho\left(F V^{-1}\right)=\max \left\{R_{H I V}, R_{T B}\right\},
$$

where $q$ indicates the spectral radius of $F V^{-1}$. By Theorem 2 [5], we obtain the following lemma.

Lemma 3. The disease-free equilibrium $P_{0}$ is locally asymptotically stable if $R_{0}<1$ and unstable if $R_{0}>1$.

\subsection{Bifurcation diagrams of the integer order model (4)}

In this subsection we show some bifurcation diagrams for the integer model (4). We use XPPAUT [6] to build the bifurcation diagrams. We vary parameters $c_{1}$, the average number of sexual partners per person and per unit time, and $b$ the tuberculosis transmission rate.

Fig. 2 shows a sketch of the bifurcation diagram for different values of $c_{1}$, the average number of sexual partners per person and per unit time. We start from a disease-free equilibrium at $c_{1} 1 \frac{1}{4}$ 0:86 and increase the value of $c_{1}$. At $c_{1} 1 / 43: 434$, there is a bifurcation point (1), at which the model bifurcates to the stable HIV endemic equilibrium. This means that increasing the number of sexual partners of susceptible individuals will, as expected, translate in new cases of HIV infections.

Fig. 3 shows the sketch of the bifurcation diagram for different values of $b$, the tuberculosis transmission rate. We start from a disease-free equilibrium and increase $b$. At $b 1 / 4$ 0:6625 (point 1), there is a bifurcation point, where the model bifurcates to the stable TB endemic equilibrium. This means that increasing the tuberculosis transmission rate will promote the appearance of new cases of TB, this is a biologically reasonable outcome. 


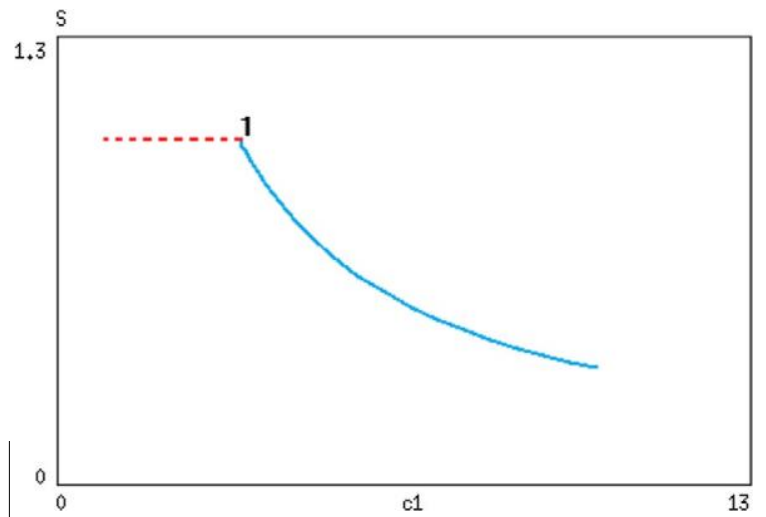

Fig. 2. Sketch of the bifurcation diagram for different values of $c_{1}$, the average number of sexual partners per person and per unit time. Remaining parameter values are given in Table 1 . Red dashed line - disease-free equilibrium, blue solid line - stable HIV endemic equilibrium. At point $1, c_{1} 1 / 43: 434$. (For interpretation of the references to color in this figure legend, the reader is referred to the web version of this article.)

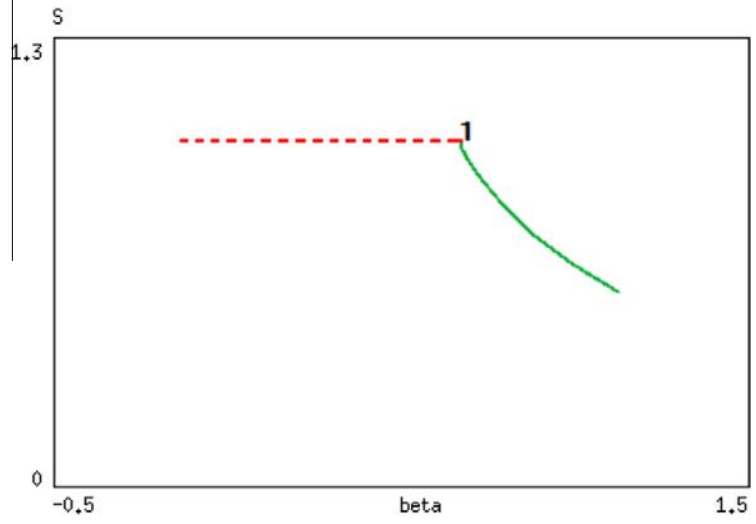

Fig. 3. Sketch of the bifurcation diagram for different values of $b$, the tuberculosis transmission rate. Remaining parameter values are given in Table 1 . Reddashed line - disease-free equilibrium, green solid line - stable TB endemic equilibrium. At point 1, b 1/4 0:6625. For more information, see text. (For interpretation of the references to color in this figure legend, the reader is referred to the web version of this article.)

Table 1

Parameters used in the numerical simulations of model (7).

\begin{tabular}{ll}
\hline Parameter & Value \\
\hline$h$ & 0.3 \\
$\mathrm{E}$ & 0.2 \\
$t$ & 0.1 \\
$b_{1}$ & 0.2 \\
$b_{3}$ & 0.8 \\
$b$ & 0.5 \\
$c 1$ & 3 \\
$p$ & 0.4 \\
$d$ & 0.6 \\
$d_{1}$ & 0.3125 \\
$d_{2}$ & 0.0625 \\
$d_{3}$ & 0.375 \\
$t_{1}$ & 0.11 \\
$t_{2}$ & 0.2 \\
\hline
\end{tabular}

\section{Numerical results}

In this section, we analyze several numerical simulations of model (7). We study the dynamical behavior of the model for

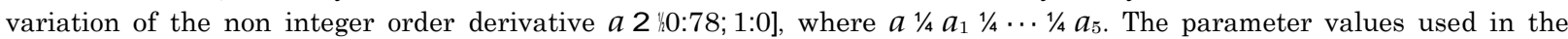




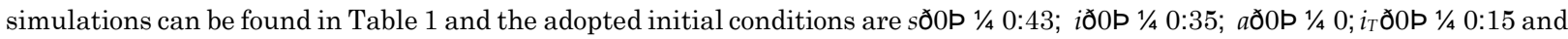
$i_{\mathrm{TH}_{0}} 1 / 4$ 0:07.

For the numerical implementation of the fractional order derivatives, a series expansion based on the Grünwald-Letnikov definition was adopted.

In Fig. 4, we plot the dynamics of the variables of system (7), for $a 2$ k0:78; $1: 0]$. We observe that, for the given parameters values and initial conditions, the model approaches asymptotically the stable disease-free equilibrium.
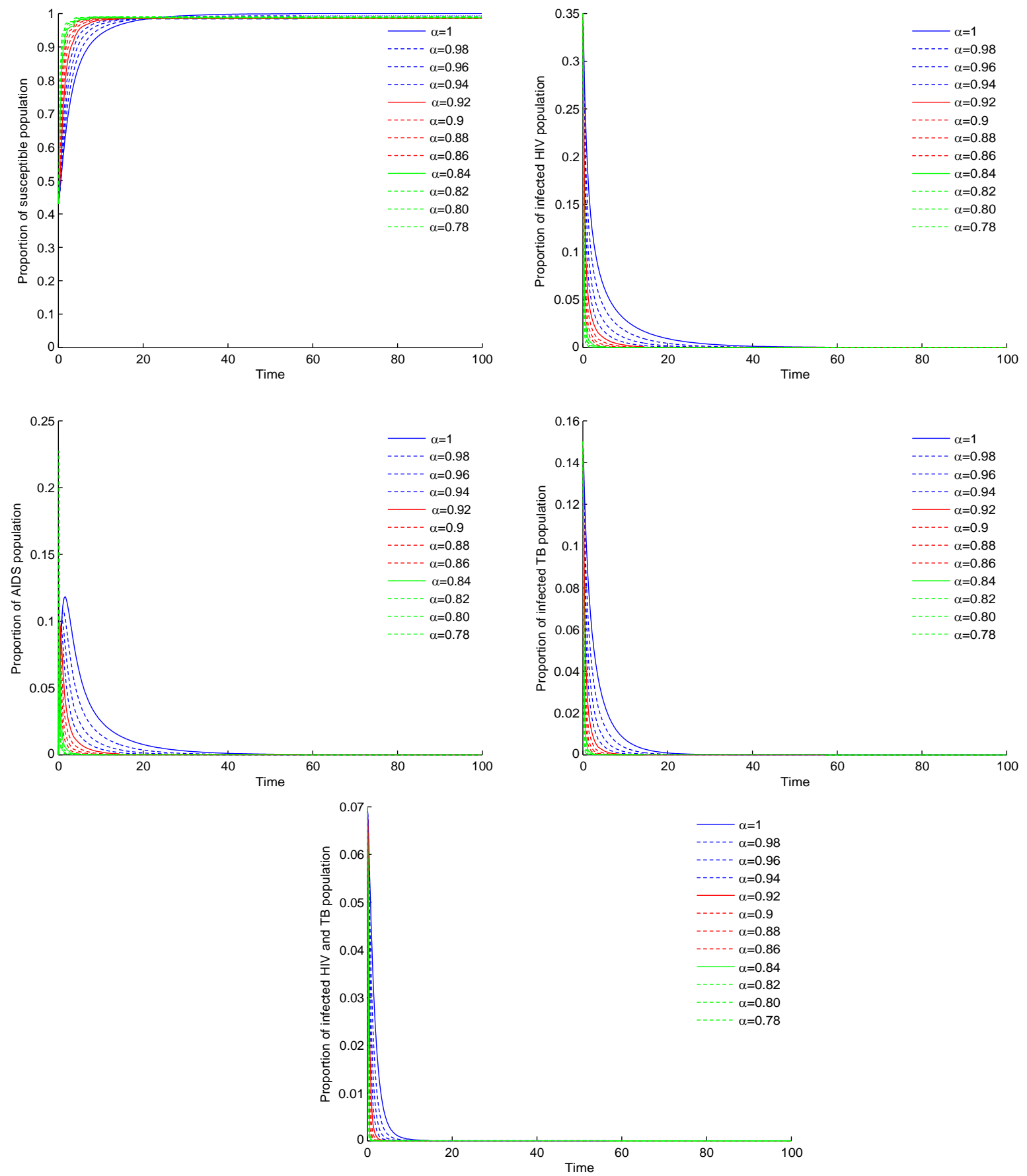

Fig. 4. Disease-free equilibrium of system (7) for variation of the order of the fractional derivative $a 2 \% 0: 78 ; 1: 0]$. Parameter values are as in Table 1 and initial conditions are as adopted. 

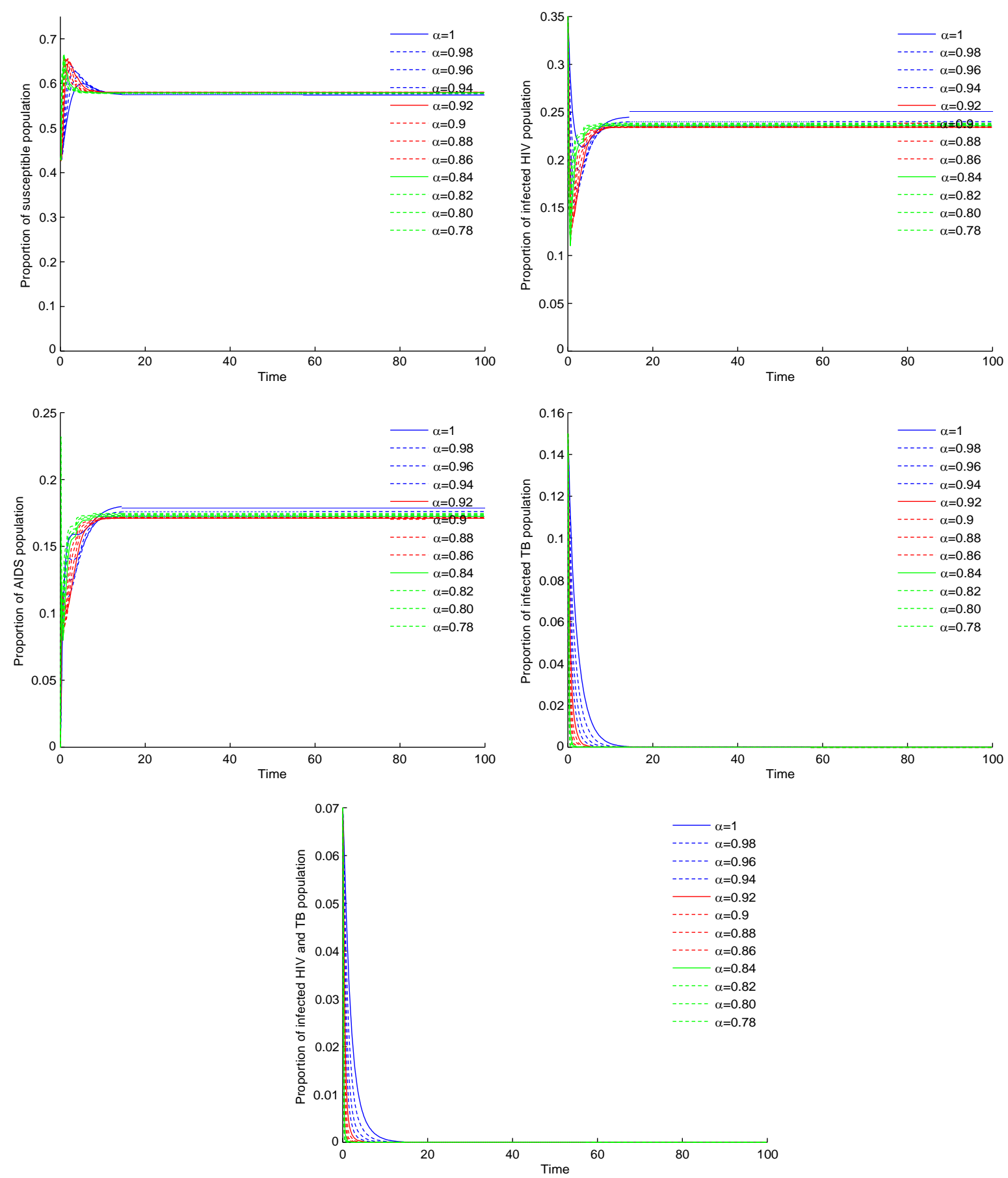

Fig. 5. HIV endemic equilibrium of system (7) for variation of the order of the fractional derivative $a 2$ : $0: 78$; $1: 0]$. Parameter values are as in Table 1 , except for $b_{1} 1 / 40: 4$, and initial conditions are as adopted.

Figs. 5 and 6, depict the dynamics of the HIV endemic equilibrium and of the TB endemic equilibrium, respectively, of system (7), for distinct values of $a 2$ 1/78; $0: 0]$. We increased the value of sexual contact rate, to $b_{1} 1 / 40: 4$ in Fig. 5 . In Fig. 6, we increased the value of the TB transmission rate to $b 1 / 40: 68$. All other parameters are as in Table 1 .

Fig. 7, shows the dynamics of the variables of system (7), for $a 20: 78 ; 1: 0]$. We observe that, for the given parameters values and initial conditions, the model approaches asymptotically two endemic equilibria. The full endemic equilibrium is obtained for 0:80<a $61: 0$. Then there is a bifurcation (change of dynamical behavior) and for $a \mathbf{6} 0: 80$, the model switches to a stable HIV endemic equilibrium. 

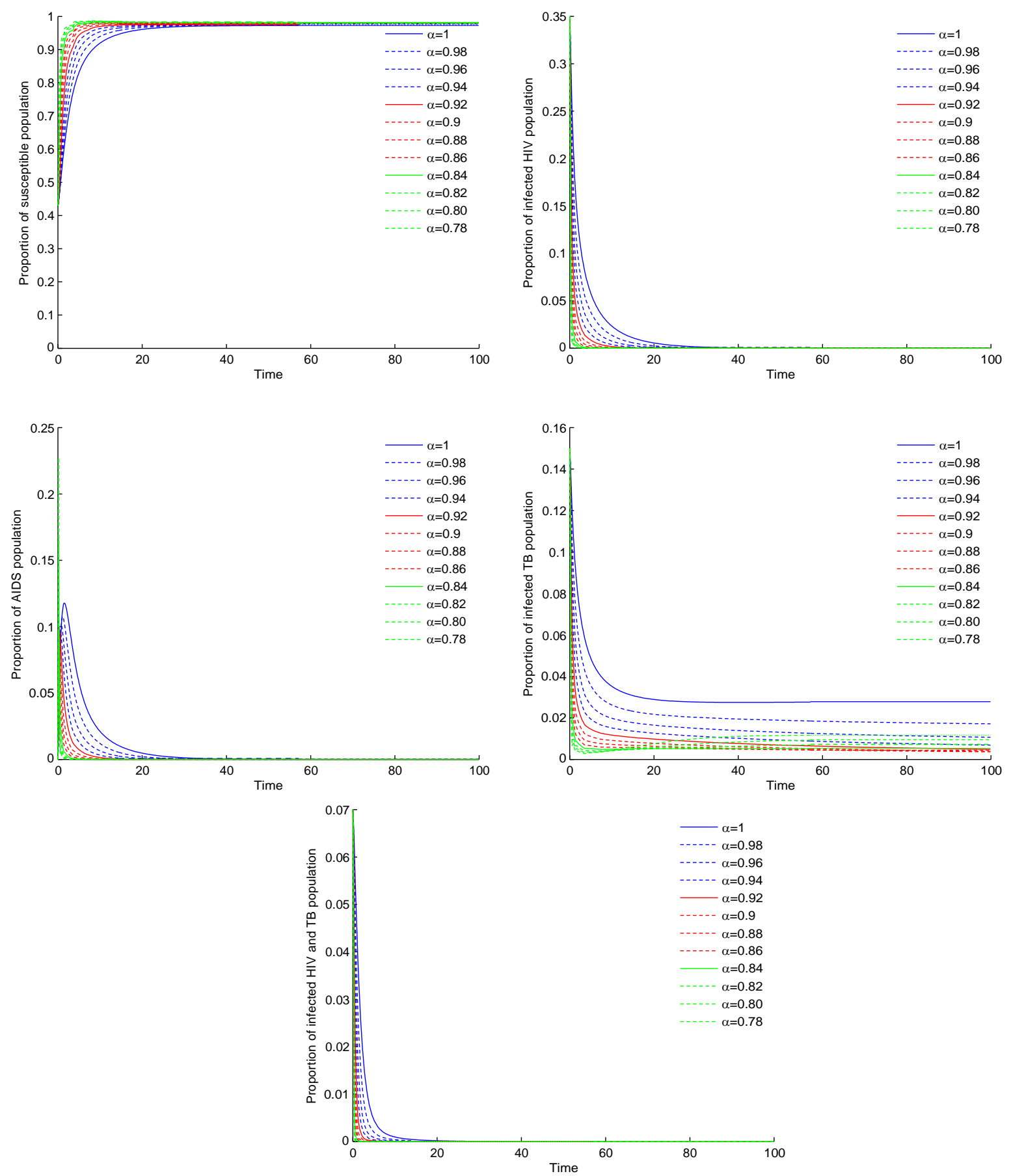

Fig. 6. TB endemic equilibrium of system (7) for variation of the order of the fractional derivative $a 2$ [0:78; $1: 0]$. Parameter values are as in Table 1 , except for $b^{1 / 4} 0: 68$, and initial conditions are as adopted.

We observe that the results of the fractional order version of the model reveal that the dynamical evolution produces new types of transients. Namely, we observe fastest transients for smaller values of $a 2$ k:78; $1: 0]$. This is a typical behavior of the fractional order systems. Another interesting fact is that the order of the fractional order, $a$, seems to play the role of a 'bifurcation parameter'. In fact, Fig. 7 depicts a transition from a stable full endemic equilibrium to a stable HIV endemic equilibrium. Biologically this would mean that people recover from TB and that the two deadly diseases cannot coexist for longer periods in the population [8]. 

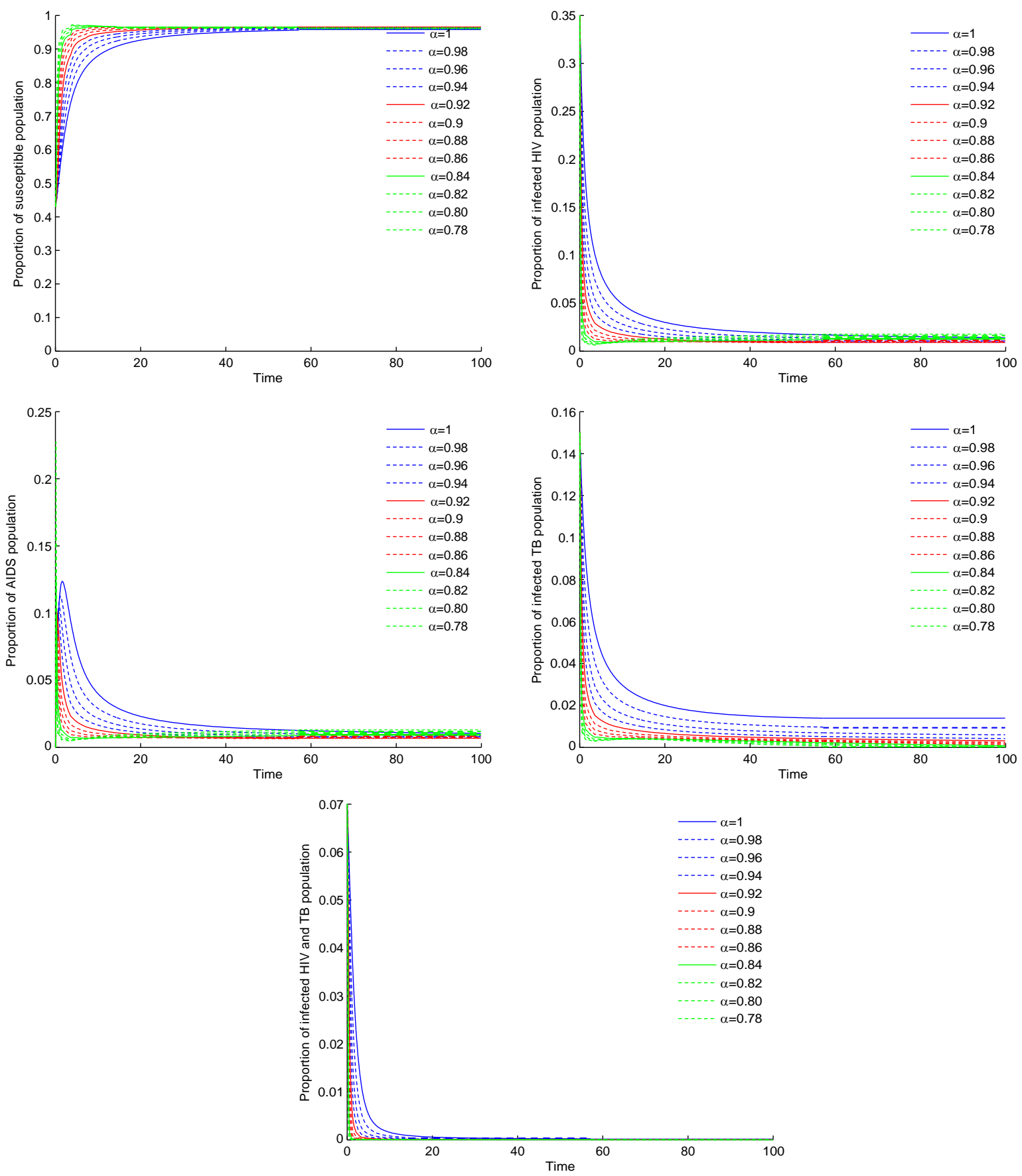

Fig. 7. Endemic equilibria of system (7) for variation of the order of the fractional derivative $a 20: 78 ; 1: 0]$. Parameter values are as in Table 1 , except for $b_{1} 1 / 40: 24$ and $b 1 / 40: 69$, and initial conditions are as adopted.

\section{Conclusion}

We proposed integer order and fractional order versions of an HIV and TB coinfection model. The model includes vertical transmission for HIV and treatment for both diseases. The reproduction number and the stability of the disease-free equilibrium were computed for the integer order model. Moreover, some bifurcation diagrams were drawn, using XPPAUT. Simulations of the fractional order model were carried out, for distinct values of the order of the fractional derivative. The results unravel new types of transients and an interesting fact, namely, the order of the fractional derivative might be seen as a 'bifurcation parameter' for the model. We conclude that the dynamics of the integer and the fractional order versions of 
the model are very rich and that together these versions may provide a better understanding of the dynamics of HIV and TB coinfection.

\section{Acknowledgments}

Authors wish to thank Fundação Gulbenkian, through Prémio Gulbenkian de Apoio à Investigação 2003, and the Polytechnic of Porto, through the PAPRE Programa de Apoio à Publicação em Revistas Científicas de Elevada Qualidade for financial support. The authors were partially funded by the European Regional Development Fund through the program COMPETE and by the Portuguese Government through the FCT - Fundação para a Ciência e a Tecnologia under the project PEst-C/MAT/UI0144/ 2013.

\section{References}

[1] D. Baleanu, About fractional quantization and fractional variational principles, Commun. Nonlinear Sci. Numer. Simul. 14 (6) (2009) $2520-2523$.

[2] M. Caputo, F. Mainardi, A new dissipation model based on memory mechanism, Pure Appl. Geophys. 91 (8) (1971) $134-147$.

[3] Center of Disease Control (CDC). <http://www.cdc.gov/HIV>.

[4] N. Dalal, D. Greenhalgh, X. Mao, A stochastic model of AIDS and condom use, J. Math. Anal. Appl. 325 (2007) $36-53$.

[5] P. Driessche, P. Watmough, Reproduction numbers and sub-threshold endemic equilibria for compartmental models of disease transmission, Math. Biosci. 180 (2002) 29-48.

[6] B. Ermentrout, XPPAUT ${ }^{\circledR}$ - The differential equations tool, version 5.98, 2006. <http://www.math.pitt.edu/r-bard/xpp/xpp.html>

[7] European Centre for Disease Prevention and Control (ECDC), Annual epidemiological report Reporting on 2011 surveillance data and 2012 epidemic intelligence data, 2013. (Report coordinated by Graham Fraser, Frantiska Hrubá, Chantal Quinten and Catalin Albu.).

[8] S. Gakkhar, N. Chavda, A dynamical model for HIV-TB co-infection, Appl. Math. Comput. 280 (2012) 9261-9270.

[9] F. Mainardi, Fractional relaxation-oscillation and fractional diffusion-wave phenomena, Chaos Solitons Fractals 7 (1996) $1461-1477$.

[10] K.S. Miller, B. Ross, An Introduction to the Fractional Calculus and Fractional Differential Equations, John Wiley and Sons, 1993.

[11] S. Momani, Z. Odibat, Analytical approach to linear fractional partial differential equations arising in fluid mechanics, Phys. Lett. A 355 (4-5) (2006) $271-279$.

[12] R. Naresh, A. Tripath, Modelling and analysis of HIV-TB coinfection in a variable size population, Math. Model. Anal. 10 (2005) 275-286.

[13] R. Nigmatullin, D. Baleanu, Is it possible to derive newtonian equations of motion with memory?, Int J. Theor. Phys. 49 (2010) $701-708$.

[14] K.B. Oldham, Fractional differential equations in electrochemistry, Adv. Eng. Softw. 41 (1) (2010) 9-12.

[15] K.B. Oldham, J. Spanier, The Fractional Calculus: Theory and Application of Differentiation and Integration to Arbitrary Order, Academic Press, 1974.

[16] C.M.A. Pinto, J.A. Tenreiro Machado, Complex order biped rhythms, Int. J. Bifurcation Chaos 21 (10) (2011) 3053-3061.

[17] C.M.A. Pinto, J.A. Tenreiro Machado, Fractional central pattern generators for bipedal locomotion, Nonlinear Dyn. 620 (1-2) (2010) 27-37.

[18] C.M.A. Pinto, J.A. Tenreiro Machado, Fractional model for malaria disease, in: Proceedings of the ASME 2013 International Design Engineering Technical Conferences \& Computers and Information in Engineering Conference IDETC/CIE 01/2013, 2013.

[19] C.M.A. Pinto, J.A. Tenreiro Machado, Fractional model for malaria transmission under control strategies, Comput. Math. Appl. 66 (5) (2013) 908-916.

[20] C.M.A. Pinto, J.A. Tenreiro Machado, Complex-order forced van der Pol oscillator, J. Vib. Control 18 (14) (2012) $2201-2209$.

[21] C.M.A. Pinto, J.A. Tenreiro Machado, Complex order van der Pol oscillator, Nonlinear Dyn. 65 (3) (2011) $247-254$.

[22] I. Podlubny, Fractional-order systems and PID-controllers, IEEE Trans. Autom. Control 44 (1) (1999) $208-213$.

[23] S.G. Samko, A.A. Kilbas, O.I. Marichev, Fractional Integrals and Derivatives: Theory and Applications, Gordon and Breach Science Publishers, 1993.

[24] V.E. Tarasov, Fractional Dynamics: Applications of Fractional Calculus to Dynamics of Particles, Fields and Media, Springer, New York, 2010.

[25] J.A. Tenreiro Machado, Analysis and design of fractional-order digital control systems, Syst. Anal. Model. Simul. 27 (2-3) (1997) 107-122.

[26] J.A. Tenreiro Machado, Fractional derivatives: probability interpretation and frequency response of rational approximations, Commun. Nonlinear Sci. Numer. Simul. 14 (9-10) (2009) 3492-3497.

[27] A.S. Waziri, E.S. Massawe, O.D. Makinde, Mathematical modelling of HIV/AIDS dynamics with treatment and vertical transmission, Appl. Math. 2 (2012) 77-89.

[28] World Health Organization TB/HIV, 2012. <http://www.who.int/gho/publications/world_health_statistics>. 